TRANSACTIONS OF THE

AMERICAN MATHEMATICAL SOCIETY

Volume 351, Number 9, Pages 3659-3672

$\mathrm{S} 0002-9947(99) 02139-\mathrm{X}$

Article electronically published on May 3, 1999

\title{
ON VASSILIEV KNOT INVARIANTS INDUCED FROM FINITE TYPE 3-MANIFOLD INVARIANTS
}

\author{
MATT GREENWOOD AND XIAO-SONG LIN
}

\begin{abstract}
We prove that the knot invariant induced by a $\mathbb{Z}$-homology 3sphere invariant of order $\leq k$ in Ohtsuki's sense, where $k \geq 4$, is of order $\leq k-2$. The method developed in our computation shows that there is no $\mathbb{Z}$-homology 3-sphere invariant of order 5 .
\end{abstract}

\section{INTRODUCTION}

The theory of finite type knot invariants is by now well developed (see $[\mathrm{V}],[\mathrm{Gu}]$, $[\mathrm{B}-\mathrm{L}],[\mathrm{K}]$ and $[\mathrm{BN} 1])$. It provides a unified approach to various quantum knot invariants (Jones, HOMFLY and Kauffman polynomials, etc.). Such an analogous unified approach to quantum 3-manifold invariants is not available at present.

In [O1], Ohtsuki reorganized quantum $S U(2)$ invariants $\tau_{r}(M)$ for a $\mathbb{Z}$-homology 3 -sphere $M$ into a formal power series

$$
\tau(M)=\sum_{n=0}^{\infty} \lambda_{n}(M)(t-1)^{n} .
$$

In order to find a characterization of the invariants $\lambda_{n}(M)$, Ohtsuki introduced the notion of finite type $\mathbb{Z}$-homology 3 -sphere invariants. Precisely, let $L$ be an algebraically split link with framings \pm 1 on each component in a $\mathbb{Z}$-homology 3 sphere $M$ and we will denote by $M_{L}$ the $\mathbb{Z}$-homology 3 -sphere obtained by surgery on $L$. Let $\lambda$ be a $\mathbb{Z}$-homology 3 -sphere invariant, then $\lambda$ is said to be a finite type invariant of order $\leq k$ if the following sum vanishes for all choices of $M$ and $L$ such that $\# L>k$ :

$$
\sum_{L^{\prime} \subset L}(-1)^{\# L^{\prime}} \lambda\left(M_{L^{\prime}}\right)=0 .
$$

Here $\# L$ is the number of components of the link $L$.

Let $\mathcal{O}_{k}$ be the vector space of all finite type invariants of $\mathbb{Z}$-homology 3 -spheres of order $\leq k$. The main result of [O2] is that $\operatorname{dim}\left(\mathcal{O}_{k}\right)<\infty$. Moreover, it is proved that the " $k$-th difference" of a finite type $\mathbb{Z}$-homology 3 -sphere invariant of order $k$ is determined by its "weight" on the set of graphs of $k$ edges such that (1) the valence at each vertex is either 1 or 3 and (2) every edge connects two distinct vertices.

Received by the editors June 29, 1995 and, in revised form May 2, 1997.

1991 Mathematics Subject Classification. Primary 57M25.

The second author is supported in part by NSF. 
In [G], among other things, Garoufalidis proved that $\mathcal{O}_{4}=\mathcal{O}_{3}$. He also observed that every $\lambda \in \mathcal{O}_{k}$ determines a finite type knot invariant $v_{\lambda}$ of order $\leq k-1$ by

$$
v_{\lambda}(K)=\lambda\left(S^{3}\right)-\lambda\left(S_{K}^{3}\right)
$$

for every knot $K$ in $S^{3}$ with +1 framing. A way of calculating the weight system of $v_{\lambda}$ was also described in $[\mathrm{G}]$. Garoufalidis suggested that it might be interesting to study these induced knot invariants, although it was also observed that the presence of Kirby moves may restrict the induced knot invariants considerably.

In this paper, we examine closely Ohtsuki's proof of the finiteness of $\operatorname{dim}\left(\mathcal{O}_{k}\right)$. This leads to one of our main results showing that the order of an induced knot invariant is, in most cases, actually one less than what it was supposed to be $a$ priori.

Theorem 1.1. Let $v_{\lambda}$ be the knot invariant induced by $\lambda \in \mathcal{O}_{k}$ with $k \geq 4$. Then $v_{\lambda}$ is of order at most $k-2$.

Our method also leads to the determination of $\mathbb{Z}$-homology 3 -sphere invariants of order $\leq 5$.

Theorem 1.2. $\mathcal{O}_{5}=\mathcal{O}_{4}$.

In [R1], [R2] Rozansky has shown, using physical predictions of the asymptotic behavior of Witten's Chern-Simons path integral over trivial connections, that Ohtsuki's invariant $\lambda_{n}$ is of order $3 n$ and he further conjectured that $\mathcal{O}_{3 n}=\mathcal{O}_{3 n+1}=$ $\mathcal{O}_{3 n+2}$. The results of this paper certainly point in this direction.

Let $\lambda_{\mathrm{C}}$ be the Casson invariant and 1 be the constant invariant whose value on each $\mathbb{Z}$-homology 3 -sphere is 1 . It is known that $\left\{1, \lambda_{\mathrm{C}}\right\}$ is a basis for $\mathcal{O}_{3}$ (see $[\mathrm{O} 2]$ and $[\mathrm{G}]$ ). Let $v_{\mathrm{C}}$ be the knot invariant induced by $\lambda_{\mathrm{C}}$. It is known that $v_{\mathrm{C}}$ is of order 2. To illustrate Theorem 1.1, we may consider the order $6 \mathbb{Z}$-homology 3 -sphere invariant $\lambda_{\mathrm{C}}^{2}$ whose induced knot invariant $v_{\mathrm{C}}^{2}$ is of order 4 . Probably also motivated by this example, Garoufalidis asked whether the knot invariant induced by a $\mathbb{Z}$-homology 3 -sphere invariant of order $\leq 3 n$ is actually of order $\leq 2 n$. The answer to this question remains open.

Remark. Since the submission of this paper, much progress has been made on the structure of the filtered space $\mathcal{O}=\bigcup \mathcal{O}_{k}$. For example, it is now known that $\mathcal{O}_{3 n}=$ $\mathcal{O}_{3 n+1}=\mathcal{O}_{3 n+2}$ (Garoufalidis-Levine) and that $\lambda \in \mathcal{O}_{3 n}$ implies that $v_{\lambda}$ is of order at most $2 n$ (Habegger and Garoufalidis-Levine). We will refer the reader to [Li] for a survey of, as well as references to, these and other developments. Nevertheless, we hope that this paper will still provide an interesting and illuminating piece in the puzzle assembled rapidly in the last year through the efforts of many people.

Acknowledgment. We appreciate greatly the referee's patience and persistence on the accuracy of the exposition.

\section{Definitions AND Basic PROPERTiEs.}

In this section we will first recall a few definitions and lemmas of Ohtsuki [O2]. Then we will give a proof of Ohtsuki's finiteness theorem using the notion of surgery classification of links in $S^{3}$. Our proof here will have the same essential ingredients as in Ohtsuki's proof. But we do think that our exposition is clearer than that of Ohtsuki's. We single out a key step in our argument, the proof of Theorem 2.2, in 

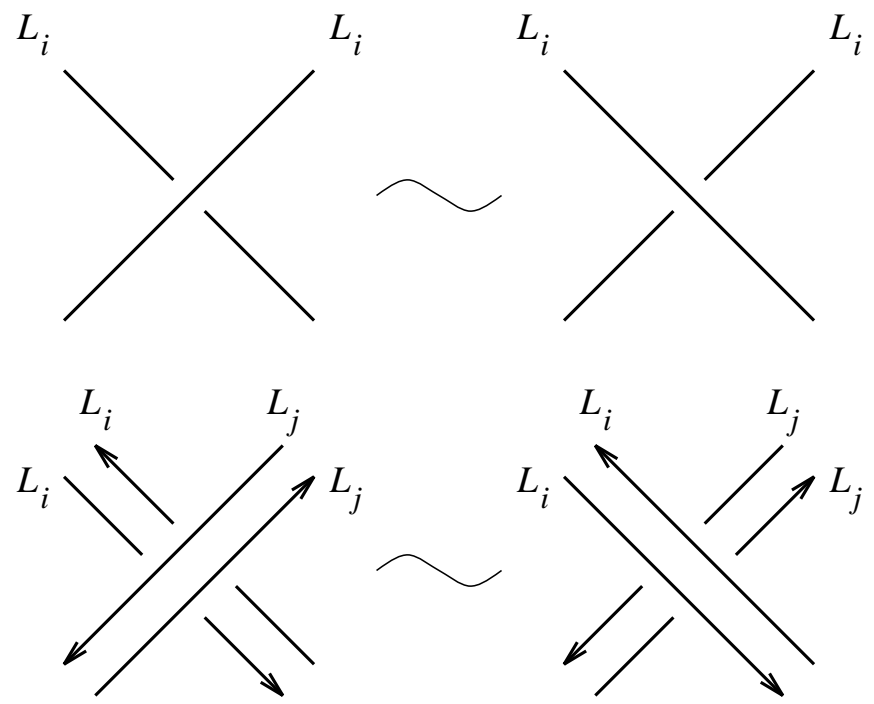

Figure 2.1. Local moves on ASL's.

the next section since the same method will be used in the proof of Theorem 1.1 in the last section.

An algebraically split link (ASL) $L$ in a $\mathbb{Z}$-homology 3 -sphere $M$ is a link with unoriented and unordered components such that all of its linking numbers are zero. Henceforth, all 3-manifolds will be $\mathbb{Z}$-homology 3 -spheres and all links will be \pm 1 framed ASL's.

Let $\mathcal{I}$ be the set of homeomorphism classes of $\mathbb{Z}$-homology 3-spheres. Let $\mathcal{O}$ be the vector space over $\mathbb{C}$ with $\mathcal{I}$ as a basis. Define an element $(M, L)$ in $\mathcal{O}$ by

$$
(M, L)=\sum_{L^{\prime} \subset L}(-1)^{\# L^{\prime}} M_{L^{\prime}}
$$

where $L^{\prime}$ runs through all sublinks (including the empty sublink) of $L$. Let $\mathcal{O}_{k}$ be the subspace of $\mathcal{O}$ spanned by $(M, L)$ for all $\mathbb{Z}$-homology 3 -spheres $M$ and all \pm 1 -framed links $L$ such that $\# L=k+1$. The space $\mathcal{O}$ has a natural stratification:

$$
\mathcal{O}=\mathcal{O}_{-1} \supset \mathcal{O}_{0} \supset \mathcal{O}_{1} \supset \cdots \supset \mathcal{O}_{k-1} \supset \mathcal{O}_{k} \supset \cdots .
$$

For $A, B \in \mathcal{O}_{k-1}$, we denote $A \sim B$ if $A-B \in \mathcal{O}_{k}$. We have the following basic lemmas from $[\mathrm{O} 2]$.

Lemma 2.1. For any $(M, L) \in \mathcal{O}_{k-1}$, there is a link $J$ in $S^{3}$ with $\# J=k$ such that $(M, L) \sim\left(S^{3}, J\right)$.

Lemma 2.2. $\left(S^{3}, L\right) \sim\left(S^{3}, J\right)$ if $J$ can be obtained from $L$ by either of the local moves shown in Figure 2.1 where $L_{i}$ and $L_{j}$ may be different components of $L$. In the second local move, we may orient components of $L$ in any favorable way.

Lemma 2.3. Let $L$ and $J$ be the same link in $S^{3}$ with different \pm 1 framings. Let $s(L)$ be the product of framings of $L$. Then $s(L)\left(S^{3}, L\right) \sim s(J)\left(S^{3}, J\right)$.

From these lemmas, we may think of $\mathcal{O}_{k-1} / \mathcal{O}_{k}$ as spanned by equivalence classes of ASL's in $S^{3}$ with $k$ components under the equivalence relations depicted in 
Figure 2.1, where an ASL $L$ is regarded as an element $\left(S^{3}, L\right) \in \mathcal{O}_{k-1}$ with the understanding that the framings of $L$ are all +1 . Now it seems to be the right moment to recall the notion of surgery classification of links in $S^{3}$ here.

Definition 2.1. A surgery modification on an ASL $L$ in $S^{3}$ is first to have a disk $B$ in $S^{3}$ intersecting $L$ only in its interior with zero algebraic intersection number. Then, perform a \pm 1 surgery on $\partial B$ which will change $L$ to another ASL $J$ in $S^{3}$. Two ASL's in $S^{3}$ are surgery equivalent if one of them can be changed to another by a finite sequence of surgery modifications.

Notice that Figure 2.1 also serves as an illustration of a surgery modification. Thus, we have

Lemma 2.4. If $L$ and $J$ are surgery equivalent $A S L$ 's with $k$ components, then $\left(S^{3}, L\right) \sim\left(S^{3}, J\right)$.

To arrive at the finiteness of $\operatorname{dim}\left(\mathcal{O}_{k-1} / \mathcal{O}_{k}\right)$, we recall the following theorem of Levine $[\mathrm{L}]$.

Theorem 2.1. Surgery equivalence classes of ASL's with oriented and ordered components are classified by Milnor's triple $\mu$-invariants.

We may now think of a surgery equivalence class of ASL's with $k$ components as a collection of integers $\left\{\mu\left(i_{1} i_{2} i_{3}\right)\right\}$ where $i_{1}, i_{2}, i_{3}$ are distinct indices among $1, \ldots, k$. They satisfy the following relations:

$$
\left\{\begin{array}{l}
\mu\left(i_{1} i_{2} i_{3}\right)=\mu\left(i_{2} i_{3} i_{1}\right)=\mu\left(i_{3} i_{1} i_{2}\right), \\
\mu\left(i_{1} i_{2} i_{3}\right)=-\mu\left(i_{2} i_{1} i_{3}\right) .
\end{array}\right.
$$

Taking advantage of being in the vector space $\mathcal{O}_{k-1} / \mathcal{O}_{k}$, each collection of integers $\left\{\mu\left(i_{1} i_{2} i_{3}\right)\right\}$ will be further simplified in the following theorem. This theorem will imply the finiteness of $\operatorname{dim}\left(\mathcal{O}_{k-1} / \mathcal{O}_{k}\right)$.

Theorem 2.2. In $\mathcal{O}_{k-1} / \mathcal{O}_{k}$, an ASL representing $\left\{\mu\left(i_{1} i_{2} i_{3}\right)\right\}$ can be expressed as a linear combination of ASL's whose collections of triple $\mu$-invariants satisfy the following conditions:

1. each $\mu\left(i_{1} i_{2} i_{3}\right)$ is either 0 or 1 for certain fixed cyclic order of indices;

2. each index $i$ appears in at most two non-zero $\mu\left(i_{1} i_{2} i_{3}\right)$ 's.

Now collections $\left\{\mu\left(i_{1} i_{2} i_{3}\right)\right\}$ of 0 's and 1 's, where $i_{1}, i_{2}, i_{3}$ are distinct indices among $1, \ldots, k$ with a fixed cyclic ordering are in 1-1 correspondence with graphs with $k$ ordered edges such that

1. each vertex is of valence either 1 or 3 ;

2. each edge connects two distinct vertices; and

3. incident edges of each valance 3 vertex come equipped with a cyclic order.

Furthermore, we may forget the ordering of edges of such a graph since the components of ASL's in $\mathcal{O}_{k-1} / \mathcal{O}_{k}$ need not to be ordered. Thus, we see that $\mathcal{O}_{k-1} / \mathcal{O}_{k}$ is spanned by ASL's in 1-1 correspondence with graphs having $k$ edges and satisfying the properties (1), (2) and (3) above. In particular, we have the following corollary.

Corollary 2.1. $\operatorname{dim}\left(\mathcal{O}_{k-1} / \mathcal{O}_{k}\right)<\infty$. 


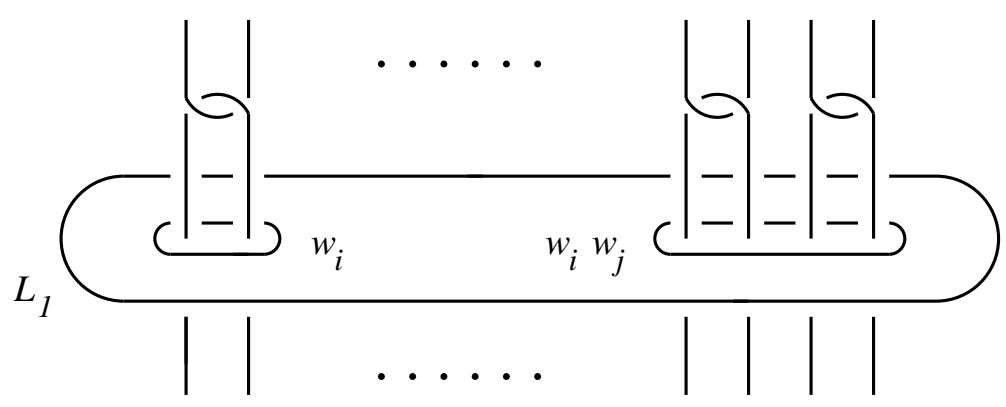

FigURE 3.1. The standard picture of a component in an ASL.

\section{The Proof of Theorem 2.2}

We start with an ASL $L$ in $S^{3}$ representing $\left\{\mu\left(i_{1} i_{2} i_{3}\right)\right\}$. We may assume that each component of $L$ is unknotted. Notice that modifying one component of $L$ will not affect the triple $\mu$-invariants not involving the index of that component. This allows us to "localize" a component of $L$ in the following way.

Pick a component, say $L_{1}$, of $L$. It bounds a disk $B$. Other components of $L$ will intersect $B$ in its interior only and the algebraic intersection numbers are all zero. Thus, we may pair the intersection points so that each pair consisting of two points lying on the same component of $L$, one with positive intersection number and the other with negative intersection number. On the disk $B$, we draw small circles $w_{1}, w_{2}, \ldots, w_{n}$ such that

1. each of them surrounds a pair of intersection points;

2. they are all disjoint; and

3. they are not nested with each other.

We may further assume that each small circle $w_{i}$ represents a simple commutator [ $\left.m_{i_{1}}, m_{i_{2}}\right]$, where $m_{i_{1}}$ and $m_{i_{2}}$ are meridians of the $i_{1}$-th and $i_{2}$-th components, respectively, with $i_{1} \neq i_{2}$. To see this, notice that we may recover the link $L$ by starting with an unlink and performing "finger moves" between different components. The components corresponding to $L_{1}$ can remain untouched, but the fingers will run through the disk it bounds (which is $B$ ) many times. We have one small circle $w_{i}$ surrounding each intersection of the fingers with $B$.

Abusing the notation, we will denote by the product $w_{1} w_{2} \cdots w_{n}$ the original link $L$, by $w_{i}$ the link obtained from replacing the component $L_{1}$ by $w_{i}$, and by $w_{i} w_{j}$ the link obtained from replacing the component $L_{1}$ by a "nice" band-sum of $w_{i}$ and $w_{j}$, with $i, j$ distinct, on the disk $B$ away from other small circles. See Figure 3.1.

Lemma 3.1. In $\mathcal{O}_{k-1} / \mathcal{O}_{k}$, we have

$$
w_{1} w_{2} \cdots w_{n}=\sum_{1 \leq i<j \leq n} w_{i} w_{j}-(n-2) \sum_{1 \leq i \leq n} w_{i} .
$$

Proof. In Figures 3.2 and 3.3, we see local pictures of +1 -framed ASL's with $k$ components. Two basic relations in $\mathcal{O}_{k-1} / \mathcal{O}_{k}$, which are consequences of Lemma 2.2, are shown in Figure 3.2. The lemma is then proved inductively following Figure 3.3. 

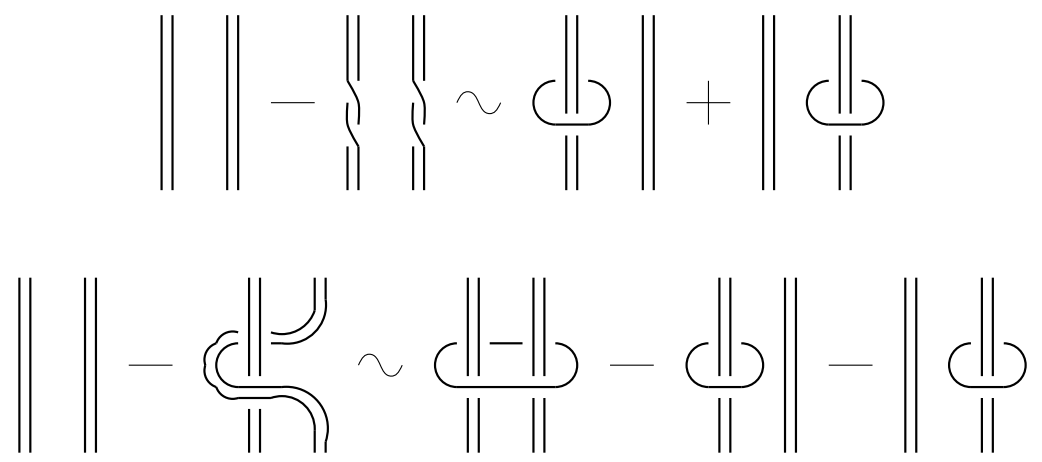

FigurE 3.2. The basic relations in $\mathcal{O}_{k-1} / \mathcal{O}_{k}$.

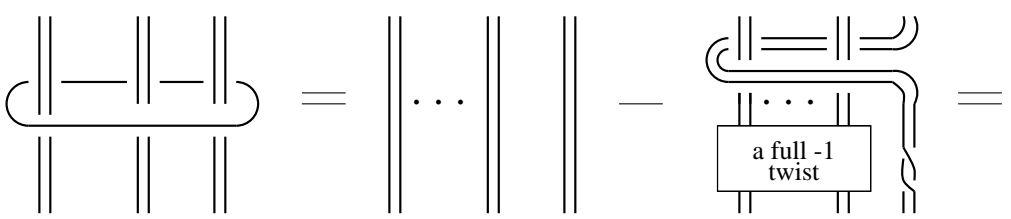

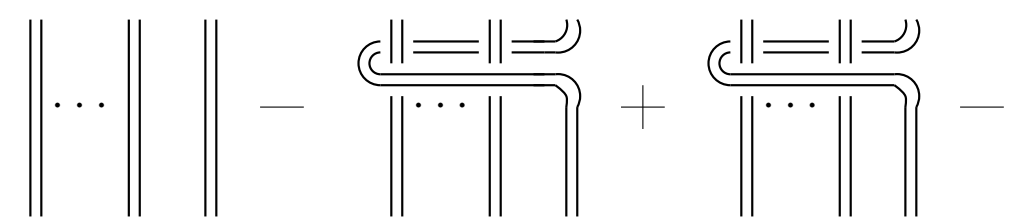

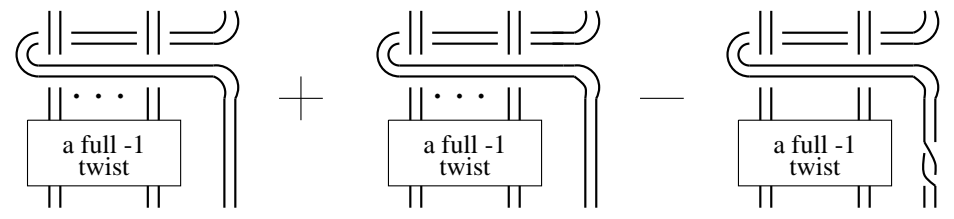

Figure 3.3. Prove Lemma 3.1 inductively.

There are three differences in the last expression in Figure 3.3. The first difference is, using the second basic relation $n-1$ times,

$$
\sum_{1 \leq i \leq n-1} w_{i} w_{n}-\sum_{1 \leq i \leq n-1} w_{i}-(n-1) w_{n} .
$$

The second difference is the ASL obtained by replacing the component $L_{1}$ with $w_{1} \cdots w_{n-1}$, which equals to, inductively,

$$
\sum_{1 \leq i<j \leq n-1} w_{i} w_{j}-(n-3) \sum_{1 \leq i \leq n-1} w_{i}
$$

Finally, the third difference is simply $w_{n}$. Putting these three differences together, we get the conclusion of Lemma 3.1. 


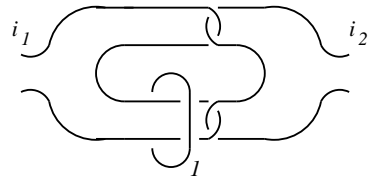

(a)

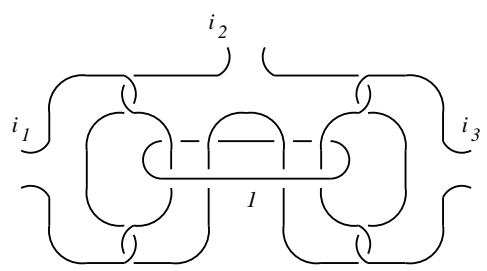

(c)

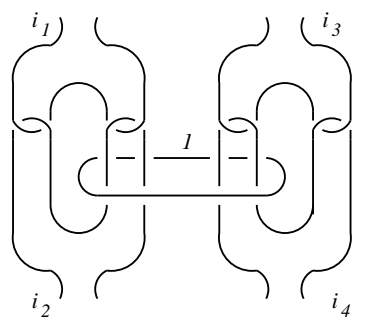

(e)

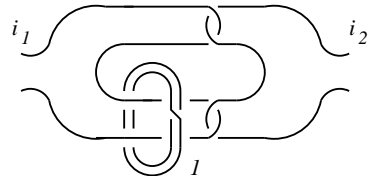

(b)

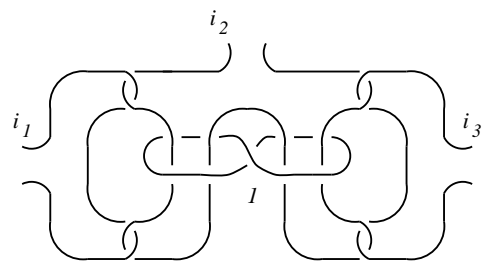

(d)

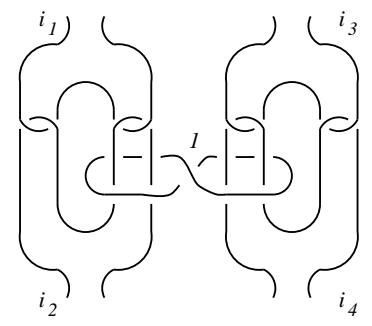

(f)

FigURE 3.4. Localization of a component.

By Lemma 3.1, we only need to understand to what extent the component $L_{1}$ in each link $w_{i}$ or $w_{i} w_{j}, i \neq j$, is "localized".

For the link $w_{i}$, there is only one triple $\mu$-invariant involving the index 1 : $\mu\left(1 i_{1} i_{2}\right)= \pm 1$. Locally, it can be realized as in Figure 3.4 (a).

For the link $w_{i} w_{j}, i \neq j$, we need to consider several cases separately.

Case 1: No non-zero triple $\mu$-invariants involving the index 1 . In this case, $w_{i} w_{j} \equiv 1$ modulo commutators of weight $\geq 3$. So $w_{i} w_{j}=0$ in $\mathcal{O}_{k-1} / \mathcal{O}_{k}$.

Case 2: Only one non-zero triple $\mu$-invariants involving the index 1: $\mu\left(1 i_{1} i_{2}\right)= \pm 2$. Locally, it can be realized as in Figure 3.4 (b).

Case 3: Two non-zero triple $\mu$-invariants involving the index 1 :

$$
\mu\left(1 i_{1} i_{2}\right) \mu\left(1 i_{2} i_{3}\right)= \pm 1
$$

with $i_{1}, i_{2}, i_{3}$ distinct. Locally, they can be realized as in Figure 3.4 (c) and (d). Case 4: Two non-zero triple $\mu$-invariants involving the index 1:

$$
\mu\left(1 i_{1} i_{2}\right) \mu\left(1 i_{3} i_{4}\right)= \pm 1
$$

with $i_{1}, i_{2}, i_{3}, i_{4}$ distinct. Locally, they can be realized as in Figure 3.4 (e) and (f).

For the link in Figure 3.4 (a), we may assume that $\mu\left(1 i_{1} i_{2}\right)=1$ since it doesn't matter how the first component is oriented in $\mathcal{O}_{k-1} / \mathcal{O}_{k}$. Similarly, in Figure 3.4 

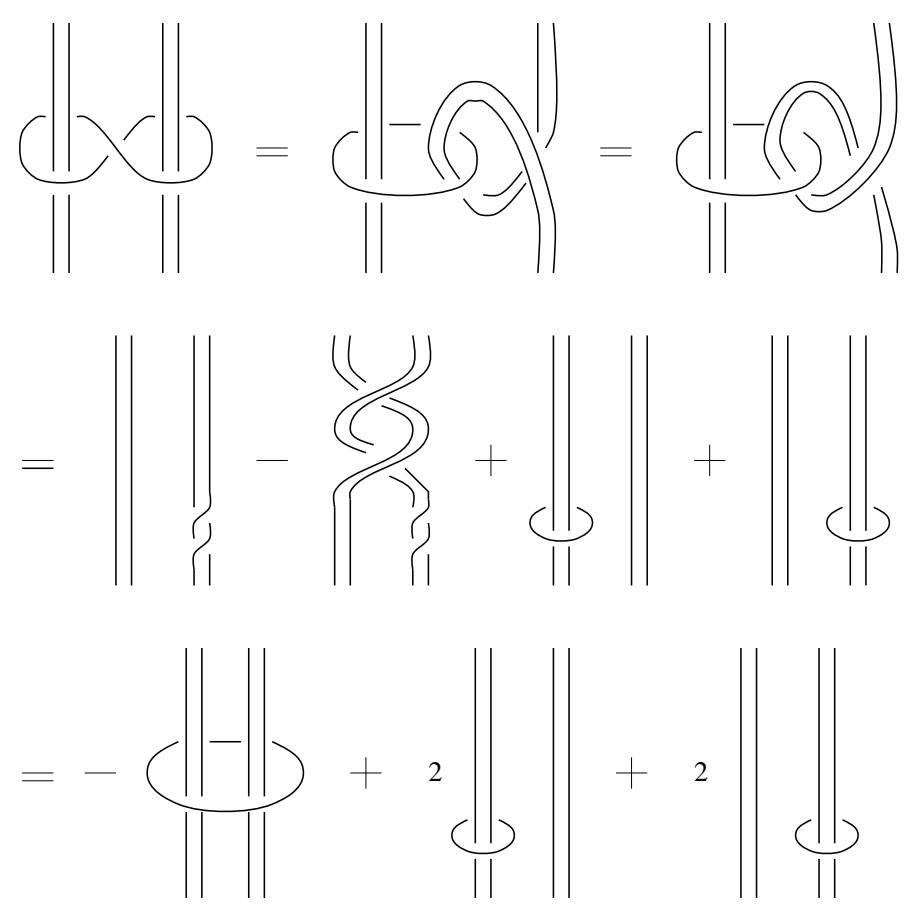

Figure 3.5. From a half twisted circle to untwisted ones.

(c) and (e), we may assume that the triple $\mu$-invariants involving the index 1 all equal to 1.

Finally, it can be seen that the links in Figure 3.4 (b), (d) and (f), viewed as elements of $\mathcal{O}_{k-1} / \mathcal{O}_{k}$, can be expressed as linear combinations of links of the form shown in Figure 3.4 (a), (c) and (e). We refer the reader to Figure 3.5, where we see that a negatively half twisted circle can be reduced to a linear combination of the same link with several different untwisted circles respectively. The first and second equalities in Figure 3.5 use the fact that we are in $\mathcal{O}_{k-1} / \mathcal{O}_{k}$ (there will be some addtional twists on the kinky bands otherewise). The third equality uses the first relation in Figure 3.2. The last equality uses the second relation in Figure 3.2 and Lemma 2.3 twice.

Thus we have achieved those two properties in Theorem 2.2 for triple $\mu$-invariants involving the index 1.

Once we have localized the first component, we proceed to localize the second component. If you look at the process of localizing a component, you will find out that the localization of the second component will affect $\mu\left(1 i_{1} i_{2}\right)$ involving the index 2 only by possibly changing a non-zero one to zero. Keep localizing each component and this completes the proof of Theorem 2.2.

To simplify the exposition, by simple graphs, we will mean graphs which satisfy

1. the valence at each vertex is either 1 or 3 ;

2. every edge connects two distinct vertices;

3 . incident edges at a valence 3 vertex have a cyclic order. 
Remark. In $\mathcal{O}_{k-1} / \mathcal{O}_{k}$, it is obvious that changing the cyclic order of incident edges at a valence 3 vertex will only change a simple graph by a sign. So we may ignore the orders at valence 3 vertices for simplicity.

\section{Induced knot invariants: The Proof of Theorem 1.1 and 1.2}

Let $\lambda$ be a $\mathbb{Z}$-homology 3 -sphere invariant. Let $L$ be an ASL in $S^{3}$ with +1 framings. We define

$$
\psi_{\lambda}(L)=\sum_{L^{\prime} \subset L}(-1)^{\# L^{\prime}} \lambda\left(S_{L^{\prime}}^{3}\right) .
$$

Lemma 4.1. A $\mathbb{Z}$-homology 3-sphere invariant $\lambda$ is of order $\leq k$ iff $\psi_{\lambda}(L)=0$ for every $A S L L$ in $S^{3}$ with $\# L>k$.

Proof. This comes from the definition (1.1) and Lemma 2.1 as well as Lemma 2.3.

Analogous to the theory of finite type knot invariants, we may think of ASL's in $S^{3}$ as "singular $\mathbb{Z}$-homology 3 -spheres" and simple graphs as "chord diagrams". For a finite type $\mathbb{Z}$-homology 3 -sphere invariant $\lambda, \psi_{\lambda}$ may then be thought of as the "derivative" of $\lambda$. The following two lemmas have their counterparts in the theory of finite type knot invariants.

Lemma 4.2. Let $\lambda$ be a $\mathbb{Z}$-homology 3-sphere invariant of order $\leq k$. Then $\lambda$ is determined by $\psi_{\lambda}$ on $A S L$ 's in $S^{3}$ with $\leq k$ components.

Proof. Let $M=S_{L}^{3}$ for a \pm 1 -framed ASL $L$ in $S^{3}$. We prove the lemma inductively on the number of components of $L$.

If $L=\emptyset$, then $M=S^{3}=\left(S^{3}, \emptyset\right)$. So the lemma holds. Now assume that $\# L=n$. Then,

$$
\lambda\left(S_{L}^{3}\right)=(-1)^{n}\left(\psi_{\lambda}(L)-\sum_{L^{\prime} \subset L, L^{\prime} \neq L}(-1)^{\# L^{\prime}} \lambda\left(S_{L^{\prime}}^{3}\right)\right) .
$$

Inductively, $\lambda\left(S_{L^{\prime}}^{3}\right)$ for $L^{\prime} \subset L, L^{\prime} \neq L$ is determined by $\psi_{\lambda}$ on ASL's in $S^{3}$ with $\leq k$ components. Since $\psi_{\lambda}(L)=0$ if $n>k$, we see that $\lambda\left(S_{L}^{3}\right)$ is also determined by $\psi_{\lambda}$ on ASL's in $S^{3}$ with $\leq k$ components.

Lemma 4.3. Let $L$ be an $A S L$ in $S^{3}$ and $O$ be an unknot separated from $L$, then $\psi_{\lambda}(L \cup O)=0$.

Proof. We have

$$
\begin{aligned}
\psi_{\lambda}(L \cup O) & =\sum_{L^{\prime} \subset L, O \notin L^{\prime}}(-1)^{\# L^{\prime}} \lambda\left(S_{L^{\prime}}^{3}\right)+\sum_{L^{\prime} \subset L, O \in L^{\prime}}(-1)^{\# L^{\prime}} \lambda\left(S_{L^{\prime}}^{3}\right) \\
& =\sum_{L^{\prime} \subset L, O \notin L^{\prime}}(-1)^{\# L^{\prime}} \lambda\left(S_{L^{\prime}}^{3}\right)+\sum_{L^{\prime} \subset L, O \notin L^{\prime}}(-1)^{\# L^{\prime}+1} \lambda\left(S_{L^{\prime} \cup O}^{3}\right) .
\end{aligned}
$$

Notice that $S_{L^{\prime} \cup O}^{3}=S_{L^{\prime}}^{3}$ for any sublink $L^{\prime}$ of $L$ such that $O \notin L^{\prime}$. Hence we have

$$
\psi_{\lambda}(L \cup O)=\sum_{L^{\prime} \subset L, O \notin L^{\prime}}(-1)^{\# L^{\prime}} \lambda\left(S_{L^{\prime}}^{3}\right)-\sum_{L^{\prime} \subset L, O \notin L^{\prime}}(-1)^{\# L^{\prime}} \lambda\left(S_{L^{\prime}}^{3}\right)=0 .
$$

This finishes the proof. 

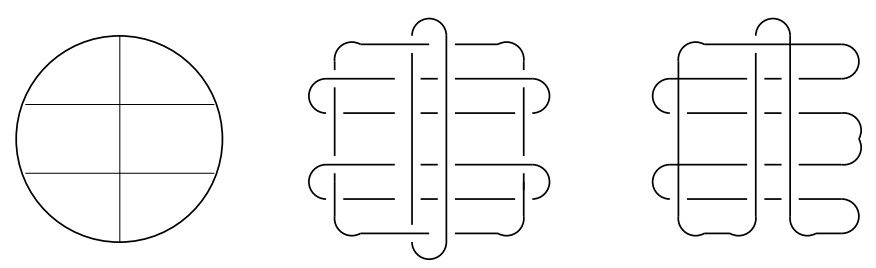

Figure 4.1. A chord diagram, its associated ASL and a singular knot respecting it.

Restricting to the case $\# L=1, \psi_{\lambda}$ becomes a knot invariant. We emphasize this situation by having a separate notation: For a knot $K$ in $S^{3}$, frame it by +1 and denote

$$
v_{\lambda}(K)=\lambda\left(S^{3}\right)-\lambda\left(S_{K}^{3}\right) .
$$

Notice that this definition of $v_{\lambda}$ differs slightly from the definition, which is given in (1.2), used in the statement of Theorem 1.1. Obviously, the conclusions of Theorem 1.1 under these two definitions are equivalent. So we may use the current definition in the proof of Theorem 1.1.

Lemma 4.4. If $\lambda$ is a $\mathbb{Z}$-homology 3-sphere invariant of order $\leq k, v_{\lambda}$ is a knot invariant of order $\leq k-1$.

Proof. This follows from Ohtsuki's first local move in Figure 2.1. For the evaluation of $v_{\lambda}$ on a knot with $k$ double points is equal to $\psi_{\lambda}(L)$ for a \pm 1 -framed ASL $L$ in $S^{3}$ with $k+1$ components, which is zero by definition of $\lambda$.

Given any chord diagram $C$ with $k-1$ chords, one may obtain an ASL with $k$ components in $S^{3}$ in the way depicted in Figure 4.1. We will first replace each chord by a thin untwisted band intersecting in its interior the outer circle at the end points of the original chord. These bands are disjoint from each other. We then take the boundaries of these bands together with the outer circle to form a ASL with $k$ components. It is a well-defined element in $\mathcal{O}_{k-1} / \mathcal{O}_{k}$ by Ohtsuki's second local move. We denote by $L(C)$ this ASL associated with a chord diagram $C$. The following lemma was observed by Garoufalidis [G] (with a sign correction noticed by the referee of this paper).

Lemma 4.5. The weight system for $v_{\lambda}$ is given by

$$
v_{\lambda}(C)=(-1)^{k-1} \psi_{\lambda}(L(C))
$$

for every chord diagram $C$ with $k-1$ chords.

Proof. Choose a "good" immersion for $C$ (which is a singular knot respecting $C$ ). This is to be done in the following way [B-L]. Pick a point on the outer circle and follow it counterclockwise. When you come to a chord, follow the chord until it is about to meet the other edge of the outer circle, go underneath it and turn back immediately to create a double point with the outer circle, then follow the chord back to where it began, and continue around the outer circle, until you have returned to the starting point. See Figure 4.1. Notice now that the immersion can always be chosen in the following way. When each double point is resolved positively, a loop is created that can be isotoped back to the outer circle. The net effect is as if that chord in the chord diagram being erased. Further, when 


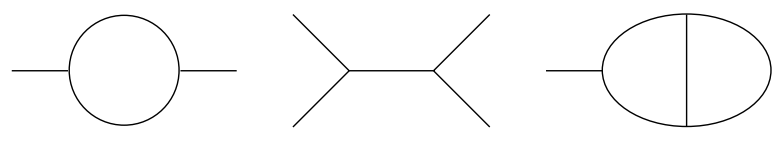

Figure 4.2. The bubble, the switch and the lollipop.

each double point is resolved negatively, the twist created in the outer circle is a negative one. The same effect can be certainly achieved by either performing or not performing (-1)-surgery on the component of $L(C)$ corresponding to that chord. Let $L_{0}$ be the outer circle of $C$ thought of as a component of $L(C)$. Then

$$
\begin{aligned}
v_{\lambda}(C) & =\sum_{L^{\prime} \subset L(C)-L_{0}}(-1)^{\# L^{\prime}} \lambda\left(S_{L^{\prime}}^{3}\right)-\sum_{L^{\prime} \subset L(C)-L_{0}}(-1)^{\# L^{\prime}} \lambda\left(S_{L^{\prime} \cup L_{0}}^{3}\right) \\
& =\sum_{L_{0} \notin L^{\prime} \subset L(C)}(-1)^{\# L^{\prime}} \lambda\left(S_{L^{\prime}}^{3}\right)+\sum_{L_{0} \in L^{\prime} \subset L(C)}(-1)^{\# L^{\prime}} \lambda\left(S_{L^{\prime}}^{3}\right) \\
& =\sum_{L^{\prime} \subset L(C)}(-1)^{\# L^{\prime}} \lambda\left(S_{L^{\prime}}^{3}\right) \\
& =(-1)^{k-1} \psi_{\lambda}(L(C)) .
\end{aligned}
$$

where all the sublinks $L^{\prime} \subset L(C)-L_{0}$ are -1 -framed. The last equality follows from Lemma 2.3.

We now use Lemma 3.1 to calculate the weight system for $v_{\lambda}$. Notice that the outer circle, call it $L_{0}$, in the ASL $L(C)$ associated with a chord diagram $C$ is quite special. If we delete $L_{0}, L(C)$ will fall apart into an unlink. Therefore, $L_{0}$ represents an element in the free group $\pi_{1}\left(S^{3} \backslash\left(L(C)-L_{0}\right)\right)$ with $k-1$ generators. Moreover, we can write out this element as a word in generators easily as follows. First, arbitrarily number the chords by $1, \ldots, k-1$, and orient them. For each chord $i$, place the symbol $i$ on the outgoing endpoint of the chord and $\bar{i}$ on the incoming endpoint. Pick a base point on the outer circle and read off the symbols encountered when traversing the circle counterclockwise. This is the word we are looking for. This word can be expressed as a product of simple commutators of the meridians of $L(C)-L_{0}$ modulo commutators of weight $\geq 3$. If the number of simple commutators is $>2$, we may use Lemma 3.1 to decompose this product of simple commutators into a sum of products of at most two simple commutators. Since there are no non-zero triple $\mu$-invariants other than these involving the index 0 (of the component $L-L_{0}$ ), such a decomposition suffices to express $L(C)$ as a sum of simple graphs.

As an example, we first reprove Garoufalidis' result in the following lemma.

Lemma 4.6. $\mathcal{O}_{4}=\mathcal{O}_{3}$.

Proof. There is only one simple graph of 4 edges, "the bubble" (see Figure 4.2), which is possibly non-zero in $\mathcal{O}_{3} / \mathcal{O}_{4}$. Suppose there is a $\mathbb{Z}$-homology 3 -sphere invariant $\lambda$ of order $\leq 4$ and let us consider the induced knot invariant of order $\leq 3$. There are two chord diagrams $C_{1}$ and $C_{2}$ with 3 chords which may have nonzero weights for an invariant of order 3. These are shown in Figure 4.3. The 4-term relation in the theory of finite type knot invariants implies that $v_{\lambda}\left(C_{2}\right)=2 v_{\lambda}\left(C_{1}\right)$. Thus, we must have

$$
\psi_{\lambda}\left(L\left(C_{2}\right)\right)=2 \psi_{\lambda}\left(L\left(C_{1}\right)\right) .
$$



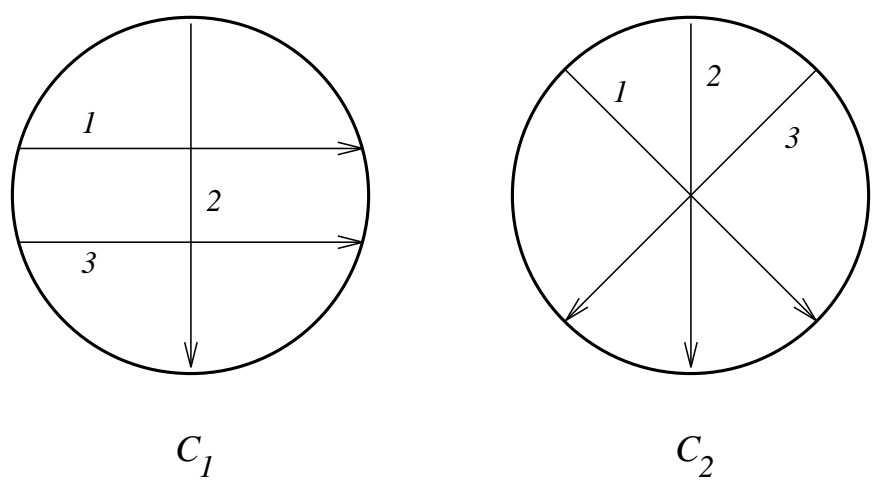

Figure 4.3. Chord diagrams with 3 chords.

Now consider their respective ASL's. It is not to hard to see that $L\left(C_{1}\right)$ can be decomposed directly as a simple graph, the bubble. Let us now decompose $L\left(C_{2}\right)$ into simple graphs. We label the chords of $C_{2}$ by $1,2,3$ and obtain the following word

$$
123 \overline{1} \overline{2} \overline{3}=[1,23][2,3] \sim[1,2][1,3][2,3]
$$

By Lemma 3.1, we can decompose this word as

$$
[1,2][1,3]+[1,2][2,3]+[1,3][2,3]-[1,2]-[1,3]-[2,3] \text {. }
$$

The simple graphs corresponding to the last three words are zero in $\mathcal{O}_{3} / \mathcal{O}_{4}$ since they all have an isolated edge. The simple graphs corresponding to the first three words are all equal to the bubble. Thus

$$
L\left(C_{2}\right)=3 L\left(C_{1}\right)
$$

in $\mathcal{O}_{3} / \mathcal{O}_{4}$. This is possible only when $\lambda=0$ on the bubble. Therefore, $\lambda$ is of order $\leq 3$.

Proof of Theorems 1.1 and 1.2. For $k=4$, the proof of Theorem 1.1 is given by Lemma 4.6. We jump to the case $k>5$ first. Let $\lambda \in \mathcal{O}_{k}$ for $k>5$. Let us evaluate the weight system of $v_{\lambda}$. For each chord diagram $C$ with $k-1>4$ chords, decompose the outer circle $L_{0}$ in $L(C)$ using Lemma 3.1 into a sum of products of at most two simple commutators. Since there are more than four chords, there will be at least one isolated edge in each of the simple graphs in the decomposition coming from Lemma 3.1 and $v_{\lambda}(C)=\lambda(L(C))=0$.

Finally, we come to the proof of Theorem 1.1 in the case when $k=5$. In this case, there are two simple graph with 5 edges, "the switch" and "the lollipop", in $\mathcal{O}_{4} / \mathcal{O}_{5}$. See Figure 4.2. It is easy to see that the lollipop has to vanish in $\mathcal{O}_{4} / \mathcal{O}_{5}$ : We may twist the tail to change the order of incident edges at a vertex, but that twist can be undone by twisting the body of the lollipop. So we only need to check the switch.

Unlike in the proof of Lemma 4.6, the induced weight system on chord diagrams with 4 chords is actually consistent as a one-dimensional vector space, whose basis element is the switch.

Nevertheless, as we will see, the local moves in Figure 2.1 put more constraints on induced knot invariants than merely the 4-term relation. Let us consider the 

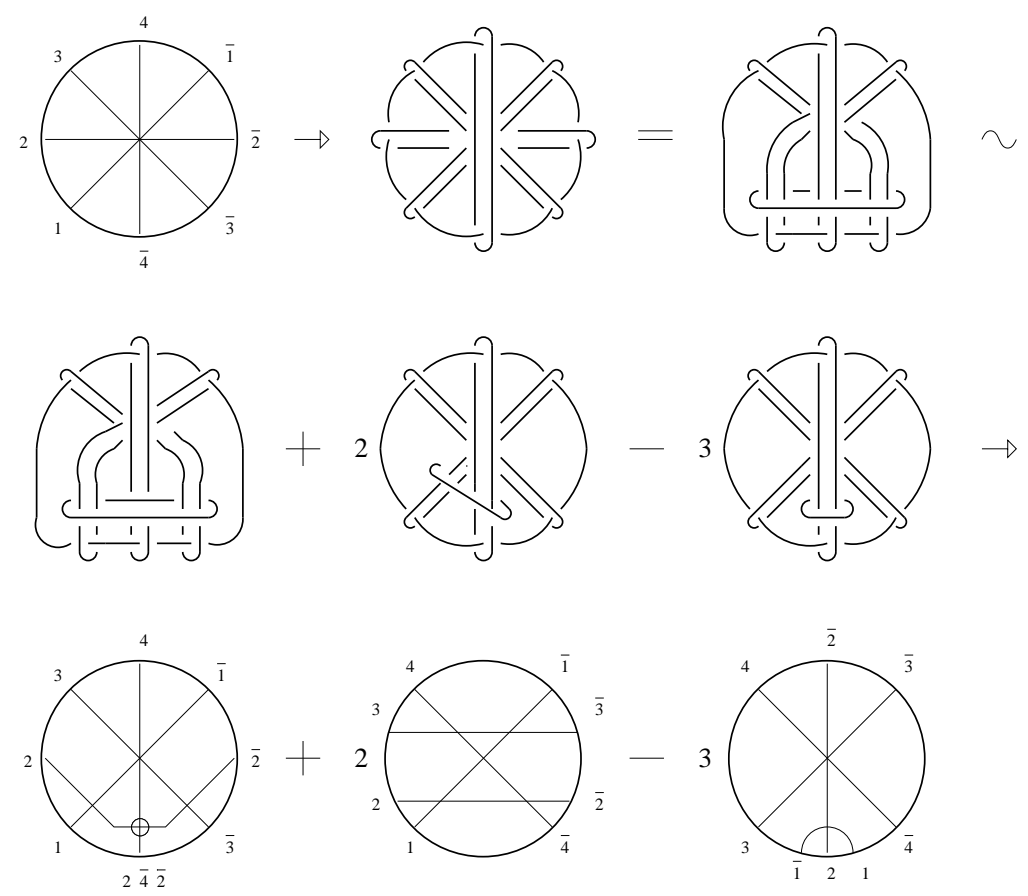

Figure 4.4. The proof of Theorem 1.1 when $k=5$ as well as Theorem 1.2.

following chord diagram $C$ with 4 chords. (Figure 4.4) Its word is given by

$$
1234 \overline{1} \overline{2} \overline{3} \overline{4}=[1,234][2,34][3,4] \sim[1,2][1,3][1,4][2,3][2,4][3,4] .
$$

Using Lemma 3.1, this evaluates to three times the switch. On the other hand, if, instead of the outer loop, we choose to apply Lemma 3.1 to one of the loops associated with a chord, we will arrive at the following diagrammatic expansion. See Figure 4.4. We first change the position of the loop associated with the chord marked by 2 so that it is in a position for us to apply Lemma 3.1. We get the next linear combination of ASL's by applying Lemma 3.1. Notice the last two ASL's in this linear combination are associated with, respectively, chord diagrams $1234 \overline{1} \overline{3} \overline{2} \overline{4}$ and $12 \overline{1} 34 \overline{2} \overline{3} \overline{4}$. They can easily be evaluated to 2 times the switch and the switch respectively. The first ASL, though, is not associated with any chord diagram. But it still has the property that when we drop the outer circle, all the other components fall apart. Thus, the same method we used to evaluate a chord diagram can be used to evaluate this ASL. We use the first diagram in the last row in Figure 4.4 to denote this ASL. The small circle at the intersection of chord 2 and chord 4 indicates that when we want to recover the original ASL, the band associated with chord 2 runs through the band associated with chord 4 . Hence we may read off the word represented by the outer circle as

$$
1234 \overline{1} \overline{2} \overline{3} 2 \overline{4} \overline{2}=[1,234][2,34][3,4][4,2]
$$

and it evaluates to 4 times the switch. Thus we get the equality that 3 times the switch is equal to 5 times the switch. This is possible only when any $\mathbb{Z}$-homology 3 -sphere invariant of order $\leq 5$ evaluates zero on the switch. We therefore have proved Theorem 1.1 in the case when $k=5$ as well as Theorem 1.2. 
The proof of Theorem 1.1 in the case when $k>5$ suggests that knots are too simple to hold up all finite type $\mathbb{Z}$-homology 3 -sphere invariants. On the other hand, Lemma 4.6 and Theorem 1.2 tell us that a detailed knowledge about induced link invariants is of great help in understanding the original 3-manifold invariants. A natural question will be: What properties characterizes the induced invariant on ASL's with two components?

\section{REFERENCES}

[BN1] D. Bar-Natan, On the Vassiliev knot invariants, Topology, 34(1995), pp. 423-471. MR 97d:57004

[B-L] J. Birman and X.-S. Lin, Knot polynomials and Vassiliev's invariants. Invent. Math., 111(1993), pp. 225-270. MR 94d:57010

[G] S. Garoufalidis, On finite type 3-Manifold Invariants I, Jour. of Knot Theory and Its Ramifications, 5(1996), pp. 441-461. MR 97j:57019

[O1] T. Ohtsuki, A polynomial invariant of integral homology 3-spheres, Math. Proc. Camb. Phil. Soc., 117(1995), pp. 83-112. MR 95i:57021

[O2] T. Ohtsuki, Finite type invariants of integral homology 3-spheres, Jour. of Knot Theory and Its Ramifications, 5(1996), pp. 101-115. MR 97i:57019

[Gu] M. Gusarov, On n-equivalence of knots and invariants of finite degree, Advances in Soviet Math. AMS, 18(1994), pp. 173-192. MR 96i:57005

[K] M. Kontsevich, Vassiliev's knot invariant, Adv. Sov. Math., 16(1993), part 2, pp. 137-150. MR 94k:57014

[L] J. Levine, Surgery on links and the $\bar{\mu}$-invariants, Topology, 26(1987), pp. 45-61. MR 88d:57005

[Li] X.-S. Lin, Finite type invariants of integral homology 3-spheres: A survey, Knot Theory, Banach Center Publication, vol. 42, Inst. of Math., Polish Acad. of Sci., Warszawa, 1998, pp. 205-220.

[R1] L. Rozansky, The trivial connection contribution to Witten's invariant and finite type invariants of rational homology spheres, preprint UMTG-182-95, q-alg/9503011

[R2] L. Rozansky, Witten's invariants of rational homology spheres at prime values of $K$ and trivial connection contribution, preprint UMTG-183-95, q-alg/9504015.

[V] V. Vassiliev, Cohomology of knot spaces, Theory of Singularities and Its Application (ed. V. Arnold), Amer. Math. Soc., 1990. MR 92a:57016

Department of Mathematics, Columbia University, New York, New York 10027

E-mail address: matt@math.columbia.edu

Department of Mathematics, University of California, Riverside, California 92521

E-mail address: xl@math.ucr.edu 